Pierre-Alain Duc, Jonathan Braine and Elias Brinks, eds.

\title{
Stellar Associations in the Tail of NGC 4038
}

\section{Saviane}

ESO, 3107 Alonso de Cordova, Vitacura - Casilla 19001, Santiago 19, Chile

J. E. Hibbard

NRAO, 520 Edgemont Road, Charlottesville, VA 22903, USA

\author{
R. M. Rich \\ Department of Physics 8 Astronomy, UCLA, Math-Sciences 8979, Los \\ Angeles, CA 90095-1562, USA
}

\begin{abstract}
We have used the Hubble Space Telescope and Wide Field Planetary Camera 2 to image the putative tidal dwarf galaxy located at the tip of the Southern tidal tail of NGC 4038/9, the Antennae. We resolve individual stars, and identify two stellar populations. Hundreds of massive stars are present, concentrated into tight $\mathrm{OB}$ associations on scales of $200 \mathrm{pc}$, with ages ranging from 2-100 Myr. An older stellar population is distributed roughly following the outer contours of the neutral hydrogen in the tidal tail; we associate these stars with material ejected from the outer disks of the two spirals. The older stellar population has a red giant branch tip at $I=26.5 \pm 0.2$ from which we derive a distance modulus $(m-M)_{0}=30.7 \pm 0.25$. The implied distance of $13.8 \pm 1.7 \mathrm{Mpc}$ is nearly a factor of two closer than commonly quoted distances for NGC $4038 / 9$. In contrast to the previously studied core of the merger, we find no super star clusters. One might conclude that SSCs require the higher pressures found in the central regions in order to form, while spontaneous star formation in the tail produces the kind of O-B star associations seen in dwarf irregular galaxies.
\end{abstract}

\section{Introduction}

Since the pioneering work of Toomre \& Toomre (1972) it has been known that dramatic tidal tails are among the most striking signs of a merger in progress. It has been suggested that the material in tidal tails might detach from the parent galaxies and collapse into gravitationally bound clumps, leading to independent objects (so-called Tidal Dwarf Galaxies or TDGs) that might form new stars and be later recognized as dwarf galaxies (Zwicky 1956; Schweizer 1978; Barnes \& Hernquist 1992; Duc et al. 1997; Duc \& Mirabel 1998). One of the best candidates for a TDG actually in the process of formation is near the end of the long, curving southern tidal tail of the well known nearby merging system NGC 4038/39, "The Antennae" (Schweizer 1978; Mirabel, Dottori \& Lutz 1992). We 
targeted this region for broadband UBVI observations with the Wide Field and Planetary Camera 2 (WFPC) on board the Hubble Space Telescope (HST). In addition to investigating the population of a TDG candidate, these observations also afford us the chance to investigate star formation taking place in an environment relatively isolated from the large scale structure of a disk, in the far outskirts of a galactic potential well.

\section{Results}

Our HST observations resolve the the stellar populations within the tail. The light of the tidal tail region imaged is dominated by bright blue stars indicative of a young stellar population. Fainter and redder stars indicative of an older population can also be seen. Selecting stars outside of star forming regions, we construct a color-magnitude diagram (CMD) for the tail. The morphology of the CMD suggests we have detected the tip of the red giant branch. If so, this

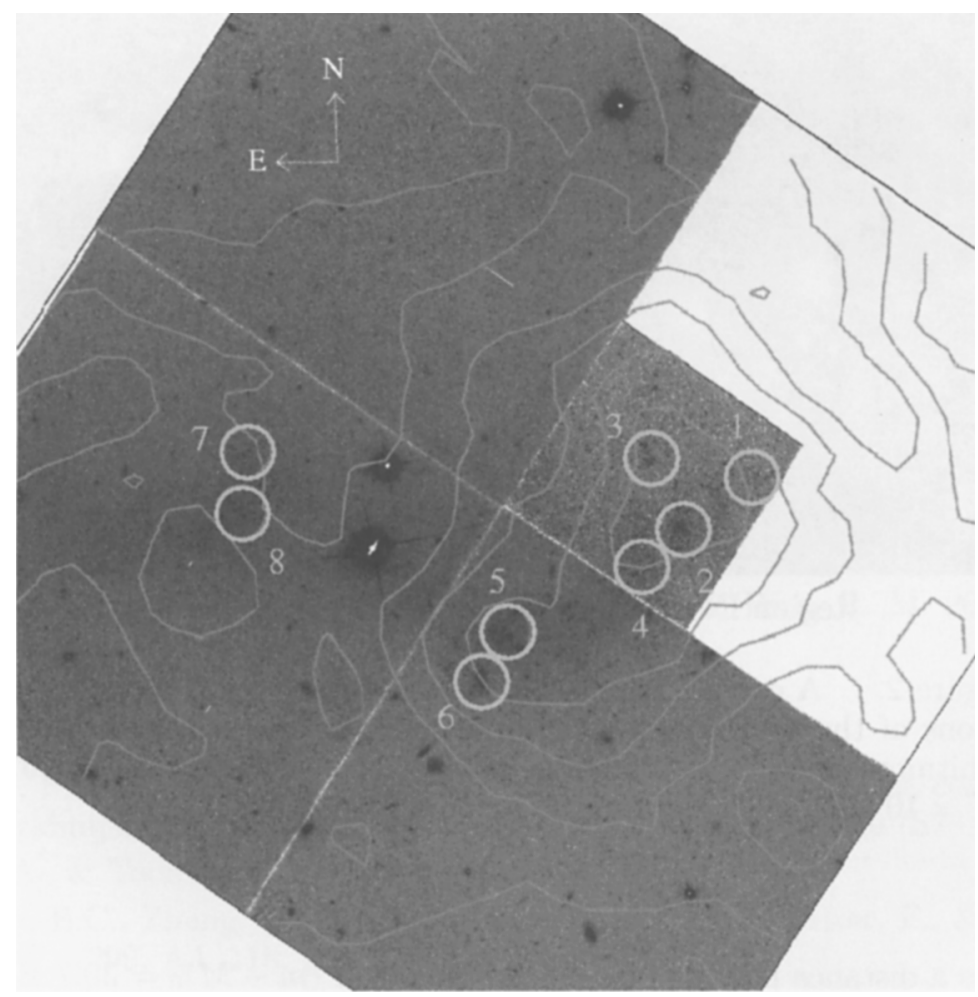

Figure 1. Greyscale representation of the F555W frame with Hi column densities from the intermediate resolution VLA datacube of Hibbard et al. (2001) and the location of the eight blue stellar associations indicated by the numbered circles with a radius of $4^{\prime \prime}$. 
Discs

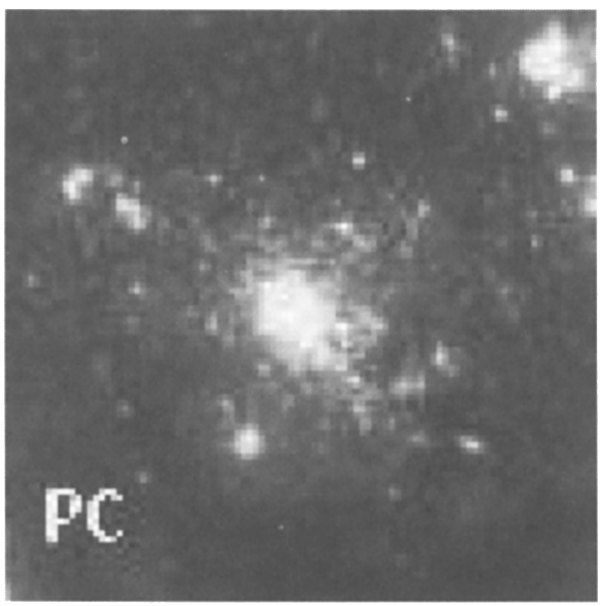

Region S

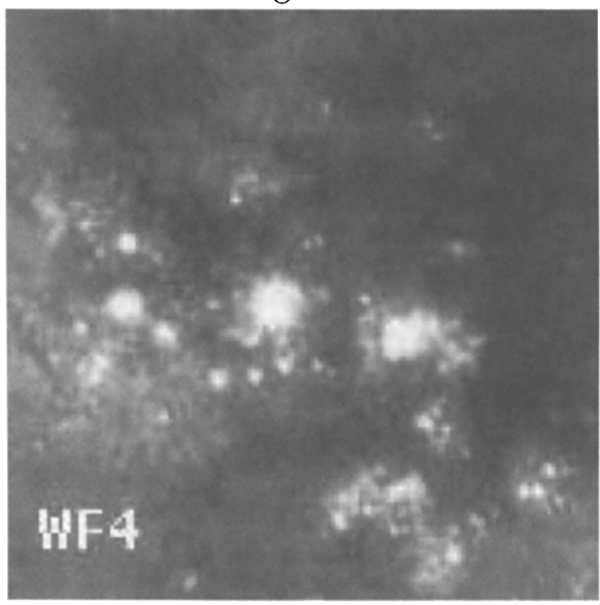

Region F
Tail

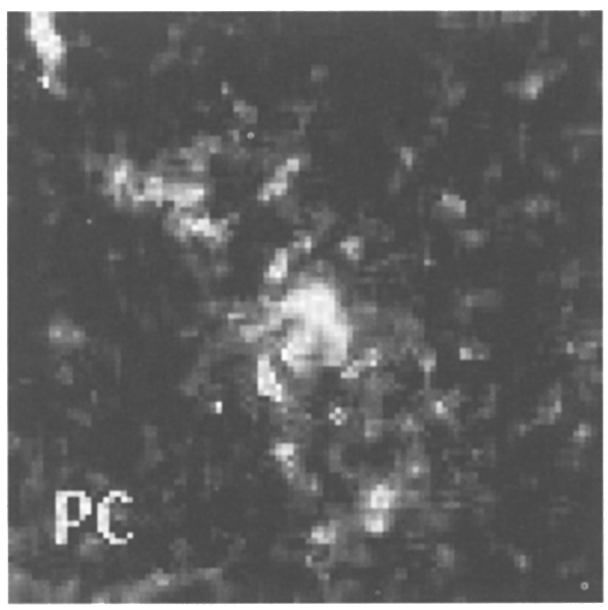

PC knot

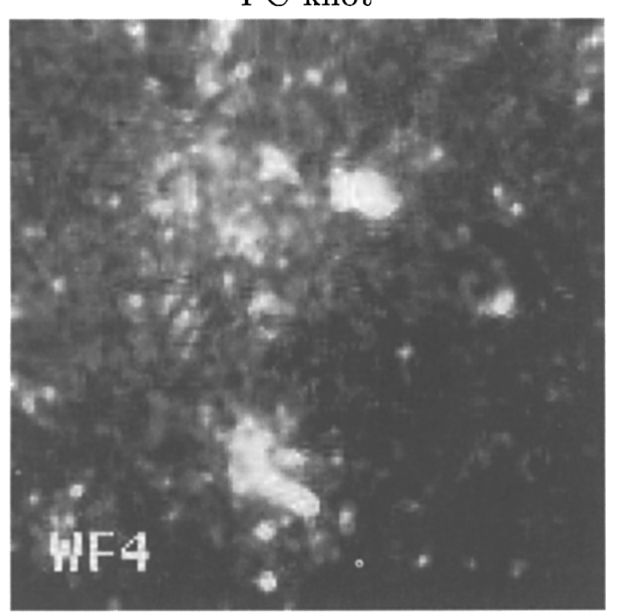

WF4 knot

Figure 2. A comparison of the visual appearance of two OB associations of the tidal tail to that of two 'super' clusters extracted from Whitmore et al. (1999). The sizes of the fields are $15^{\prime \prime} \times 15^{\prime \prime}$ and $10^{\prime \prime} \times 10^{\prime \prime}$ for the WF4 and PC images, respectively.

suggests a distance modulus for the Antennae of $(m-M)_{0}=30.7 \pm 0.25$. The reader is referred to Saviane, Hibbard \& Rich (2004, AJ, in press) for full details.

Bright red stars follow the distribution of main sequence stars, which indicates that the star formation started at least a hundred Myr ago. The faint red stars, which we identify with old stars originally in the outer disk of NGC 4038, show a broader and more uniform spatial distribution, which gives an idea of the underlying structure of the tail. 
We identify eight stellar associations within the WFPC field of view which show an overdensity of stars within a few arcseconds, and which contain stars brighter than $I_{0}=27.5$ magnitudes (i.e., younger than $32 \mathrm{Myr}$ for $Z=0.008$ and our choice of distance modulus). The location of these association with respect to both the underlying starlight and the HI gas is shown in Fig. 1. The tail stellar associations are markedly different from the so-called "Super Star Clusters" (SSCs) found within the inner regions of the merger (Whitmore et al. 1999; W99). A side-by-side comparison of the tail association with SSCs is shown in Fig. 2. The SSCs are roughly spherical and contain more than $10^{5} \mathrm{M}_{\odot}$ of stars (W99). In contrast, the associations observed in the tidal tail are irregular in shape and contain less than a hundred stars brighter than $I_{0}=28$. Even the brightest tail associations fall in the faintest bin of the W99 cluster luminosity function. It is then clear that within the tail we do not observe the kind of SSCs that have been produced in the "violent" central regions. The linear sizes look comparable to those of the central clusters, implying that the tail associations are in a much less dense environment. One might conclude that SSCs require the higher pressures found in the central regions in order to form (e.g., Jog \& Das 1996; Elmegreen \& Efremov 1997), while spontaneous star formation in the tail produces the kind of O-B star associations seen in dwarf irregular galaxies.

Acknowledgments. This work was based on observations made with the NASA/ESA Hubble Space Telescope obtained at the Space Telescope Science Institute, which is operated by the Association of Universities for Research in Astronomy, Inc., under NASA Contract NAS-5-2655. These observations were made in connection with proposal GO-6669.

\section{References}

Barnes, J.E., \& Hernquist, L. 1992, Nature, 360, 715

Duc, P.-A., Mirabel, I.F. 1998, A\&A, 333, 813

Duc, P.-A., Brinks, E., Wink, J.E., \& Mirabel, I.F. 1997, A\&A, 326, 537

Elmegreen, B. G., \& Efremov, Y. N. 1997, ApJ, 480, 235

Hibbard, J. E., van der Hulst, J. M., Barnes, J. E., \& Rich, R. M. 2001, AJ, 122,2969

Jog, C. J., \& Das, M. 1996, ApJ, 473, 797

Mirabel, I.F., Dottori, H., \& Lutz, D. 1992, A\&A, 256, L19

Schweizer, F. 1978, in Structure and Properties of Nearby Galaxies, eds. E. M. Berkhuijsen and R. Wielebinski (Dordrecht,Reidel), p. 279 (S78)

Toomre, A., \& Toomre, J. 1972, ApJ, 178, 623

Whitmore, B.C., Zhang, Q., Leitherer, C., Fall, S. M., Schweizer, F., \& Miller, B.W. 1999, AJ, 118, 1551

Zwicky, F. 1956, Ergebnisse der Exakten Naturwissenschaften, 29, 344 